progress in real time. It establishes and centralizes the continuous collection of key performance indicators and fosters accountability and transparency among cores and leadership. METHODS/ STUDY POPULATION: Using the University of Chicago's Annual Progress Report REDCap data dictionary, UI Institute for Clinical and Translational Science (ICTS) core managers convened to explore the adaptability of the reporting format for the CTSA. The team developed the more user friendly and easily accessible RPPR Reporting Tool using REDCap to better fit our CTSA. The RPPR in REDCap provides a central location to monitor the activities for each core, gather status updates, generate performance reports, and identify key performance indicators and challenges to prevent failures in the future. All data are transparent and accessible ondemand to individual core managers, evaluators, and ICTS leadership. RESULTS/ANTICIPATED RESULTS: UI's RPPR Tool has improved the compliance with ongoing monitoring and reporting of CTSA program's performance. Documenting all relevant information in a centralized space has eased the administrative and evaluation burden of preparing the RPPR. Furthermore, REDCap as a commonly used tool allows the core managers to complete this reporting with minimal guidance. This tool encourages each core to be accountable for documenting their respective progress. The transparency of the reporting allows the Co-PIs along with the leadership team to access the data at any given time to stay updated on the ICTS' overall progress and to make the appropriate improvements as needed. DISCUSSION/SIGNIFICANCE OF IMPACT: The RPPR is a required component of all CTSA grants. UI's RPPR Tool has been instrumental in comprehensively tracking progress of the ICTS and its contributions to translational research. UI is collaborating with CTSA peers to improve the RPPR Tool, so it can become an asset for any CTSA to adapt.

4051

Assessing outcomes of Miami CTSI's Mentored Career Development KL2 Program: Using bibliometric and network visualization approaches to complement traditional outcome metrics

Rosalina Das ${ }^{1}$, Jessica Diaz ${ }^{2}$, Patricia Avissar ${ }^{2}$, Tatjana Rundek ${ }^{2}$, Gwendolyn B. Scott ${ }^{2}$, Alessia Fornoni ${ }^{2}$, Jonelle E. Wright ${ }^{2}$, Sheela Dominguez ${ }^{1}$, Barry S. Issenberg ${ }^{2}$, and Ralph L. Sacco ${ }^{2}$

${ }^{1}$ University of Miami Clinical and Translational Science Institute;

${ }^{2}$ University of Miami

OBJECTIVES/GOALS: The goal of this project was to assess the scientific impact of Miami CTSI's Mentored Career Development (KL2) Program using bibliometric tools and network visualization in addition to the traditional metrics used to provide a comprehensive evaluation. METHODS/STUDY POPULATION: Scholarly productivity of KL2 scholars were tracked using REDCap. For bibliometric data analysis and visualization, publications were queried using iCite (NIH Office of Portfolio Analysis) and Web of Science database. A total of 173 publications produced by eight KL2 scholars from 2013-2018 were analyzed and categorized into pre-award, during award, and post-award periods. iCite was used to assess scientific influence and translation. Scientific networks and collaboration were visualized using VOSviewer (Centre for Science and Technology Studies, Leiden University). CTSA Common Metrics were tracked using the Results Based
Accountability framework. RESULTS/ANTICIPATED RESULTS: Albeit of modest size, the Miami CTSI's KL2 Program had significant scientific productivity and impact in its first five years. Our KL2 scholars' publications were cited twice as frequently as other papers in their fields. Further, $48 \%$ of publications post KL2 award were above the NIH 50th percentile and had higher citation impact compared to the average NIH-funded paper; $11 \%$ were in the top $10 \%$ $\mathrm{NIH}$ citation ranking. In contrast, only $20 \%$ of the publications pre-KL2 award were above the NIH 50th percentile. The program also promoted research collaboration; network visualizations indicate larger co-authorship and organization networks of KL2 scholars post-award. DISCUSSION/SIGNIFICANCE OF IMPACT: Bibliometric and data visualization approaches helped us better identify trends and gauge effectiveness of the KL2 program. These findings provided useful insight into the scientific influence and impact of our scholars' work.

4034

\section{Can Connections IN Health become a research-based model to improve health outcomes through community health coalitions?}

Lily Darbishire ${ }^{1}$, Sarah Wiehe ${ }^{2}$, and Dennis Savaiano ${ }^{1}$

${ }^{1}$ Purdue University; ${ }^{2}$ Indiana University School of Medicine

OBJECTIVES/GOALS: Connections IN Health's goal is to coordinate, integrate, and enrich health coalition work through extended connections among community and academic stakeholders within and across coalitions and geographies within Indiana. We aim to evaluate stakeholder connections to assess coalition effectiveness and the quality of partnership networks. METHODS/STUDY POPULATION: We will collect data longitudinally to evaluate Connections IN Health using a unique triangulation of effectiveness surveys, social network analysis, and health data. Cross-sectional functioning and social network analysis surveys were distributed to coalition members before the transition to Connections IN Health engagement (baseline) and will be distributed again each year thereafter to identify changes in coalition perceived effectiveness and changes in the structure/nature of partnership networks after implementation of the partnership. We plan to utilize publicly available health data to measure proximal changes in health outcomes at the neighborhood level and use Pearson's correlations to check for associations between perceived coalition effectiveness and health outcomes. RESULTS/ANTICIPATED RESULTS: We found low baseline scores in perceived effectiveness, especially in the areas of leadership, operational understanding, and satisfaction, from the coalition members. From our social network analysis, we found relatively low cohesion scores (measured as network density) among each of the coalition networks, and even lower scores for collaboration among coalition members. We expect to see positive increases in perceived coalition effectiveness, as well as an increase in the density and level of collaboration among coalition networks as Connections IN Health develops. Finally, we expect to see positive changes in proximal health outcomes associated with our measures of coalition effectiveness. DISCUSSION/SIGNIFICANCE OF IMPACT: The results of our project will be distributed back to the coalition leaders and members in order to sustain and improve the coalitions. The visualization of the coalition member's network can be used to demonstrate opportunities for enhanced partnerships and collaboration. 